University of Massachusetts Amherst

From the SelectedWorks of Qiangfei Xia

2014

\title{
Concentric rings of polystyrene and titanium dioxide nanoparticles patterned by alternating current signal guided coffee ring effect
}

Jinhua $\mathrm{Mu}$

Peng Lin

Qiangfei Xia, University of Massachusetts - Amherst 


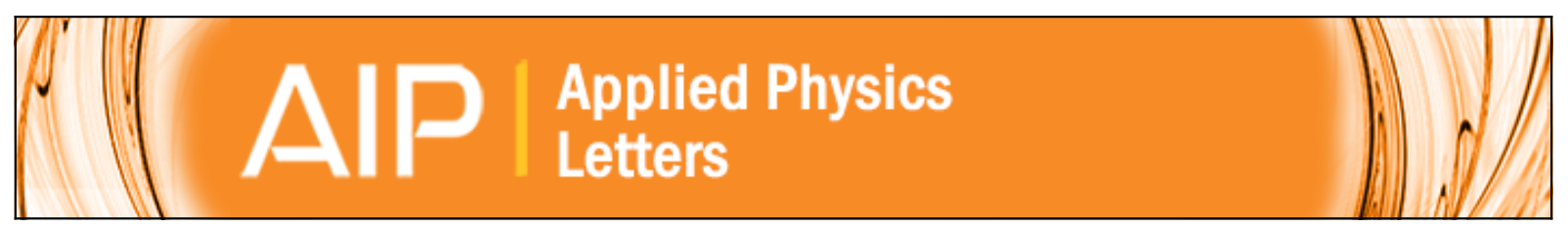

\section{Concentric rings of polystyrene and titanium dioxide nanoparticles patterned by alternating current signal guided coffee ring effect}

Jinhua Mu, Peng Lin, and Qiangfei Xia

Citation: Applied Physics Letters 104, 261601 (2014); doi: 10.1063/1.4886138

View online: http://dx.doi.org/10.1063/1.4886138

View Table of Contents: http://scitation.aip.org/content/aip/journal/apl/104/26?ver=pdfcov

Published by the AIP Publishing

\section{Articles you may be interested in}

Room temperature ferromagnetism in non-magnetic doped TiO2 nanoparticles

J. Appl. Phys. 113, 17B511 (2013); 10.1063/1.4795615

"Coffee-ring" patterns of polymer droplets

AIP Conf. Proc. 1512, 142 (2013); 10.1063/1.4790951

Patterned substrates to facilitate long-range ordering in the formation of nanoparticle monolayers by electrophoretic deposition

Appl. Phys. Lett. 101, 043117 (2012); 10.1063/1.4738988

Ring like self assembled Ni nanoparticles based biosensor for food toxin detection

Appl. Phys. Lett. 100, 093702 (2012); 10.1063/1.3690044

Nanoparticle ring formation in evaporating micron-size droplets

Appl. Phys. Lett. 84, 4774 (2004); 10.1063/1.1759378

Frustrated by

old technology?

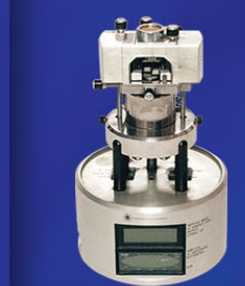

Is your AFM dead

and can't be repaired?

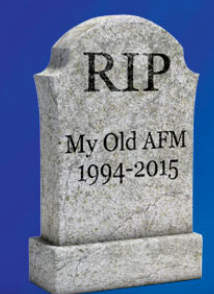

Sick of bad customer support?

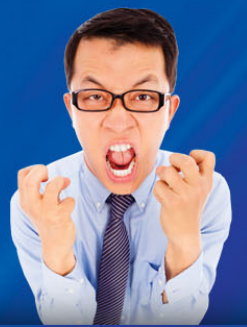

It is time to upgrade your AFM

Minimum $\$ 20,000$ trade-in discount for purchases before August 31st

Asylum Research is today's technology leader in AFM 


\title{
Concentric rings of polystyrene and titanium dioxide nanoparticles patterned by alternating current signal guided coffee ring effect
}

\author{
Jinhua Mu, ${ }^{1}$ Peng Lin, ${ }^{2}$ and Qiangfei $\mathrm{Xia}^{2, \mathrm{a})}$ \\ ${ }^{1}$ Department of Physics, University of Massachusetts, Amherst, Massachusetts 01003, USA \\ ${ }^{2}$ Nanodevices and Integrated Systems Laboratory, Department of Electrical and Computer Engineering, \\ University of Massachusetts, Amherst, Massachusetts 01003, USA
}

(Received 21 January 2014; accepted 19 June 2014; published online 30 June 2014)

\begin{abstract}
The authors studied the surface deposition of nanoparticles by introducing an alternating current (AC) signal into the millimeter-sized nanoparticle droplet. For both polystyrene (PS) in deionized (DI) water and titanium dioxide $\left(\mathrm{TiO}_{2}\right)$ in toluene, the nanoparticles self-assembled into regular concentric rings over a larger area on the substrate during the droplet drying process. The patterned area decreased, and the inter-ring spacing increased with higher $\mathrm{AC}$ frequencies for the $\mathrm{TiO}_{2} /$ toluene system, while those for the PS/DI water system only changed slightly. The frequency dependent pattern formation was interpreted by the interaction between different factors such as capillary flow and the AC signal introduced dielectrophoresis force. (C) 2014 AIP Publishing LLC.

[http://dx.doi.org/10.1063/1.4886138]
\end{abstract}

The evaporation of a drying droplet containing nonvolatile solutes leads to a ring-like pattern on the substrate, such as coffee stains that we often observe in daily life. This phenomenon is called "coffee ring effect." It has been proposed that capillary flow evoked by solvent evaporation on the droplet edge drives nonvolatile elements like suspended particles to the three phase contact line, leaving behind an irregular ring-shaped pattern with higher concentration of particles on the edge after the droplet dries up. ${ }^{1-5}$ Before this theory was proposed, a thorough mechanism had been established for the two-dimensional ordering of micro-spheres on a horizontal substrate ${ }^{6}$ and practical applications can be found in recording of colored images ${ }^{7}$ and the assembly of proteins. ${ }^{8}$ Recently, a few more applications of coffee ring effect in photonic devices, ${ }^{9}$ biology, ${ }^{10,11}$ and patterning of functional materials, such as graphene, ${ }^{12}$ have been demonstrated. The inhomogeneous distribution of the solutes in the stain, however, is not desired for applications to coating and printing. ${ }^{13}$ To effectively suppress the coffee ring effect, Marangoni effect introduced by changing the temperature profile inside the droplet ${ }^{14}$ and electrowetting effect by using direct current (DC) ${ }^{15}$ and $\mathrm{AC}$ potentials ${ }^{16}$ have been employed in previous studies. An AC signal was also used to control the evaporation of a water droplet that does not contain any nanoparticles, ${ }^{17,18}$ again utilizing the electrowetting effect. Nonetheless, neither DC nor AC signal was used to manipulate the self-assembly process of nanoparticles into regular patterns during the droplet drying process. Previous efforts towards patterning of nanoparticles through the coffee ring effect include using confined environments and sphere on a substrate to form gradient concentric ring patterns ${ }^{19}$ or spiral patterns ${ }^{20}$ from polymer solutions in a repetitive "stick-slip" manner.

In this Letter, we report on patterning of polystyrene (PS) and titanium dioxide $\left(\mathrm{TiO}_{2}\right)$ nanoparticles using electric field guided coffee ring effect. It is found that instead of

${ }^{\text {a)} E l e c t r o n i c ~ m a i l: ~ q x i a @ e c s . u m a s s . e d u ~}$ irregularly shaped coffee-stain like pattern, the PS and $\mathrm{TiO}_{2}$ particles in the droplets formed concentric rings over large area under the influence of an AC signal. The pattern geometry (such as the inter-ring distance) and patterned area are dependent on the frequency of the AC signal and the dielectric constants of the solutes and the solvents. We further explain that the pattern formation is governed by different factors such as the capillary flow and the dielectrophoresis (DEP) forces that affect the particle motion during the drying process.

In preparation of the solution, $350 \mathrm{~nm}$ PS nanoparticles (purchased from Microspheres-Nanospheres) were dissolved in deionized water in an ultrasonic bath. $100 \mathrm{~nm} \mathrm{TiO}_{2}$ nanoparticles purchased from the same company were dispersed in toluene. Concentrations of both solutions were $0.0625 \mathrm{mg} / \mathrm{ml}$. The substrates used in this study were lightly doped silicon wafers (resistivity $1-25 \Omega \cdot \mathrm{cm}$ ) with a $100 \mathrm{~nm}$ thick thermally grown silicon oxide layer on top. Arrays of $40 \mathrm{~nm}$ thick concentric Au electrodes were fabricated on the substrates, using electron beam lithography, metal evaporation, and lift-off. Each set of electrodes consisted of a $3.6 \mathrm{~mm}$-diameter ring (line-width $60 \mu \mathrm{m}$ ) and a $100 \mu \mathrm{m}$-diameter dot in the center. The substrates were then exposed to oxygen plasma briefly in a sputter $\left(10^{-7}\right.$ Torr base pressure, $20 \mathrm{sccm}$ oxygen flow rate, $0.2 \mathrm{~W} / \mathrm{cm}^{2}$ power density, $60 \mathrm{~s}$ ). To apply the electric field within the droplet, the center dot was connected to a wave-function generator that delivered the AC signals (square waveform, 0 to $5 \mathrm{~V}$ amplitude) through a tungsten probe (tip diameter $10 \mu \mathrm{m}$ ), and the outer ring was electrically grounded. The inhomogeneous electric field was applied once a millimeter-sized droplet $(1 \mu L)$ of solution was deployed on and fully spread over the whole area within the outer electrode ring. The concentric ring pattern started to form after the contact line released from the outer electrode. During the drying process, the droplet remained a spherical shape, and gravitational effect was negligible because the droplet radius $(1.8 \mathrm{~mm})$ was smaller than the values of capillary lengths for toluene and water $(1.83$ 
and $2.73 \mathrm{~mm}$ respectively, which were calculated from the equation $\lambda_{c}=\sqrt{\frac{\gamma}{\rho g}}$, where $\gamma$ is the surface tension on the airsolution interface, $\rho$ is the density of fluid, and $g$ is the magnitude of gravitational acceleration $\left.{ }^{21}\right)$. All experiments were conducted at room temperature $\left(20^{\circ} \mathrm{C}\right)$ in a clean room environment with $50 \%$ relative humidity.

The AC signal applied on the droplets significantly impacted the pattern formation during the drying process. If a droplet of PS solution was deposited on the substrate and dried naturally without any guiding condition [Fig. 1(a)], the pattern formed after evaporation was a typical "coffee ring," which had an irregular shape and a higher concentration of particles at the edge than in the center [Fig. 1(d)]. If a tungsten probe was placed in the middle of the droplet during the drying process, some concentric rings formed around the tip on the substrate after the droplet dried up [Figs. 1(b) and 1(e)], consistent with previous studies. ${ }^{19}$ However, if an AC signal $(100 \mathrm{~Hz})$ was applied to the droplet through the tungsten probe on the central electrode during the drying process, concentric stripes of nanoparticles that covered a much larger area formed on the substrate after solvent evaporation [Figs. 1(c) and 1(f)]. The patterned area in Figs. 1(e) and 1(f) were 1.06 and $6.79 \mathrm{~mm}^{2}$, respectively, a $540 \%$ increase with applying the AC signal.

The frequency of AC signal played a critical role in the pattern geometry for nanoparticles of different materials. First, the number of rings and the inter-ring spacing changed with the frequency. For example, Fig. 2 shows optical images of the dried up pattern for the $100 \mathrm{~nm} \mathrm{TiO}_{2}$ nanoparticles dispersed in toluene under AC signals at frequencies

\section{(a) No guidance}

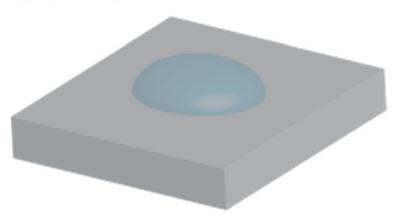

(b) Guided with probe

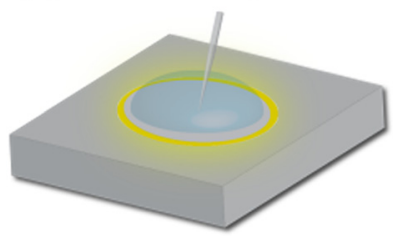

(c) Guided with AC signal

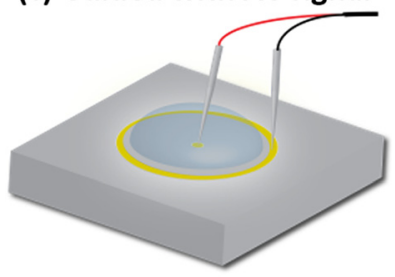

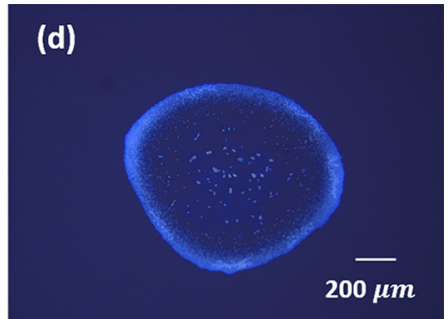
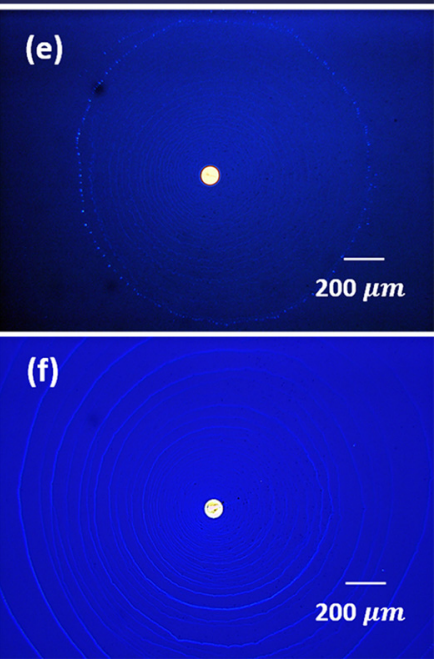

FIG. 1. Pattern formation for PS nanoparticles under three representative conditions. (a) When there is no guidance, a typical "coffee ring" formed from a $0.2 \mu L$ droplet (d). (b) With a probe in the middle of droplet, concentric rings formed over an area of $1.06 \mathrm{~mm}^{2}$ (e). (c) When an AC signal $(100 \mathrm{~Hz})$ is applied, concentric rings formed over a much larger area $(6.79$ $\left.\mathrm{mm}^{2}\right)(\mathrm{f})$. The round dots in (e) and (f) are the central electrodes.
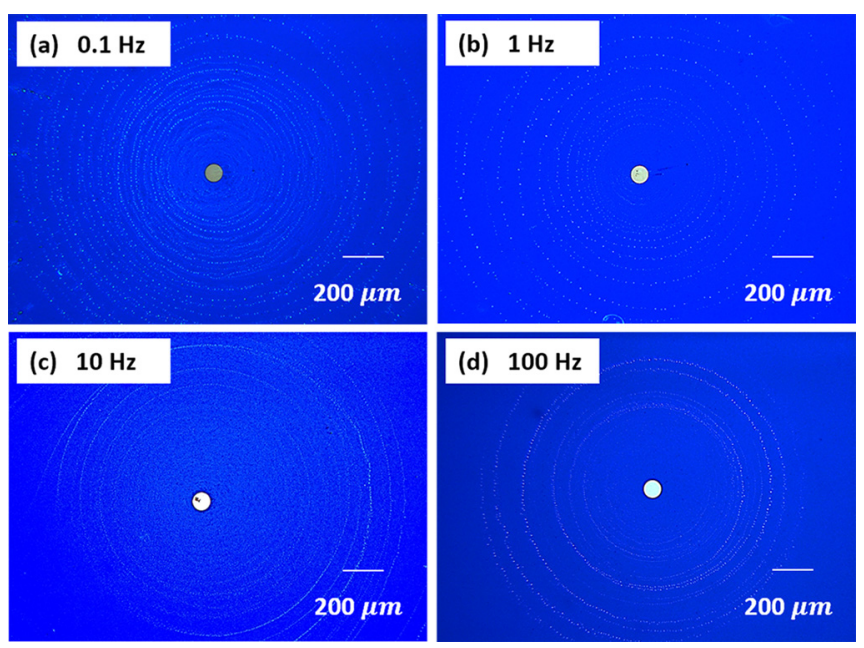

FIG. 2. Pattern formation for $\mathrm{TiO}_{2}$ nanoparticles mono-dispersed in toluene under AC frequencies of (a) $0.1 \mathrm{~Hz}$; (b) $1 \mathrm{~Hz}$; (c) $10 \mathrm{~Hz}$; (d) $100 \mathrm{~Hz}$. With higher frequencies, the patterned area becomes smaller and the inter-ring spacing becomes larger.

from 0.1 to $100 \mathrm{~Hz}$. The number of $\mathrm{TiO}_{2}$ rings decreased with increase of frequency, while the inter-ring spacing increased at the same time. However, this trend was not repeated with the PS nanoparticles dispersed in deionized (DI) water. As shown in Fig. 3, the density of rings increased slightly when the AC frequency increased from 0.1 to $100 \mathrm{~Hz}$. Accordingly, the inter-ring spacing decreased slightly, although at a nearly unnoticeable rate.

Furthermore, the patterned area on the substrates was also dependent on the frequency of the AC signal, as shown in Fig. 4(a). The patterned area was defined as the maximum region confined by the outermost concentric nanoparticle ring that was next to an irregular and non-uniform stain at the contact line of the initial droplet. This non-uniform pattern, observed for both PS and $\mathrm{TiO}_{2}$ systems, was resulted from electrowetting effect from the outer electrode. With higher AC frequency, the patterned area for $\mathrm{TiO}_{2}$ decreased (for example, from 5.73 to $2.23 \mathrm{~mm}^{2}$ when the frequency increased from 0.1 to $100 \mathrm{~Hz}$ ). While that for PS increased from 4.83 to 6.70
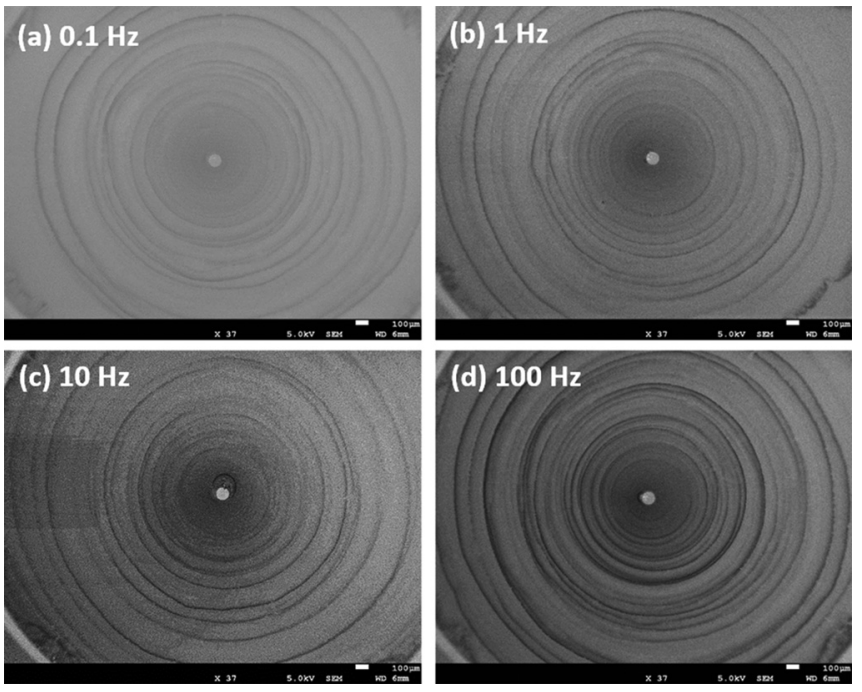

FIG. 3. SEM images of concentric PS rings left by the drying droplets at various frequencies: (a) $0.1 \mathrm{~Hz}$; (b) $1 \mathrm{~Hz}$; (c) $10 \mathrm{~Hz}$; (d) $100 \mathrm{~Hz}$. With higher frequencies, the density of rings increases slightly. Scare bars: $100 \mu \mathrm{m}$. 

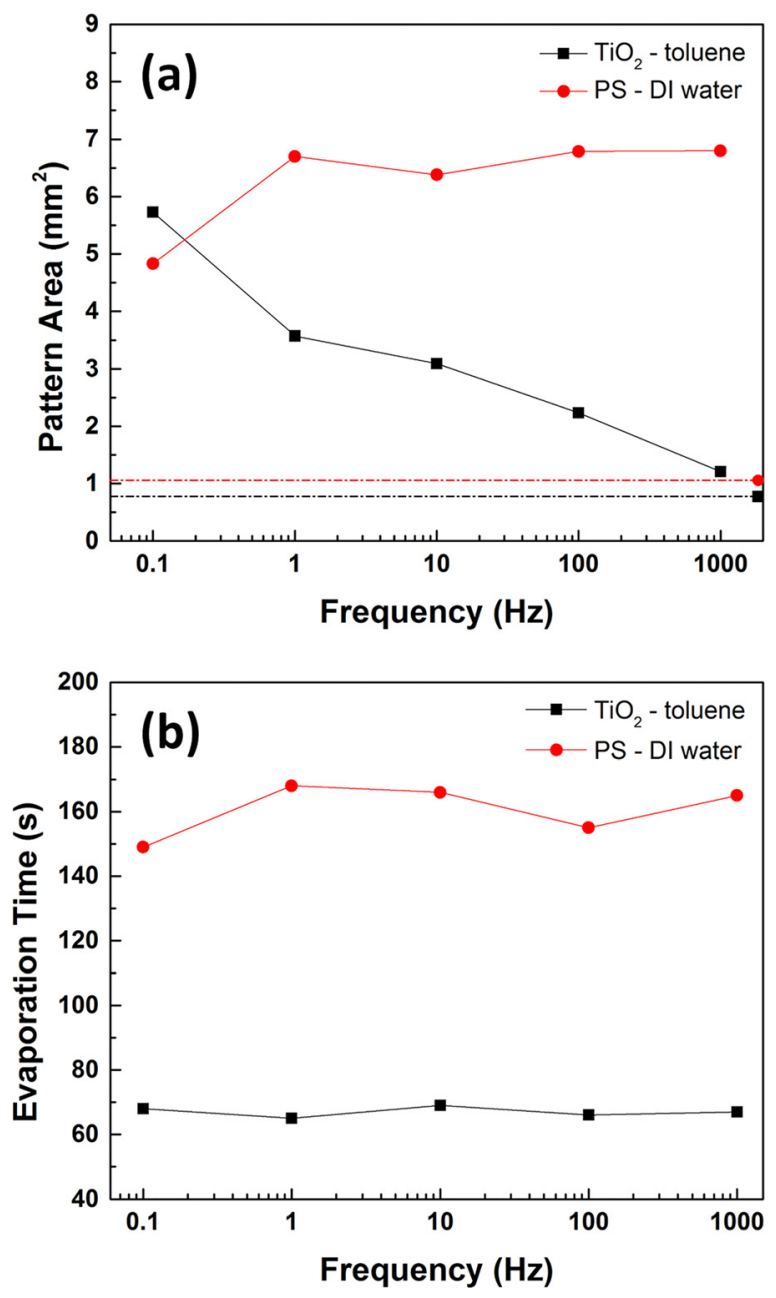

FIG. 4. (a) The pattern area as a function of AC signal frequency. When frequency increases, the patterned area for $\mathrm{TiO}_{2}$ decreases, while that for PS increases only from 0.1 to $1 \mathrm{~Hz}$ and stays unchanged afterwards. The black/ red dash lines indicate the significantly smaller patterned areas of $\mathrm{TiO}_{2} / \mathrm{PS}$ nanoparticles when the droplets were solely guided by a tungsten probe. (b) The evaporation times as a function of $\mathrm{AC}$ signal frequency. The $\mathrm{TiO}_{2} /$ toluene system is dried much faster than that of PS/DI water, while for each type of suspension, the evaporation time does not vary a lot with increasing frequencies, suggesting a nearly constant evaporation rate.

$\mathrm{mm}^{2}$ when frequency went up from 0.1 to $1 \mathrm{~Hz}$ and remained at the same level at higher frequencies. It needs to be clarified that the patterned area for $\mathrm{TiO}_{2}$ formed at $0.1 \mathrm{~Hz}$ is comparable with that defined by the outer electrodes although Fig. 2(a) only shows the central part of the pattern.

Fig. 4(b) records the average experimental evaporation times for the $1 \mu L$ suspensions under frequencies from 0.1 to $1 \mathrm{kHz}$. Again, the measurement was performed in a cleanroom environment at $20{ }^{\circ} \mathrm{C}$ and $50 \%$ relative humidity. The $\mathrm{TiO}_{2} /$ toluene solution evaporated much faster than the PS/DI water suspension. But, for each type of suspensions, we did not observe a noticeable correlation between the solvent evaporation time and the frequency of applied AC signal. Rapid evaporation could lead to the formation of periodic ring patterns, as shown in the Fig. 1(e), when AC signals were not applied. However, AC signals have helped the pattern formation over much larger areas.

In the evaporating droplet, nanoparticles under a nonuniform electric field are subjected to the DEP, a force induced by the interaction between the polarized particle (dipole) and the applied electric field. For a homogeneous spherical particle of radius $r$, the time-averaged DEP force can be written as ${ }^{22}$

$$
\mathrm{F}_{\mathrm{DEP}}=2 \pi \mathrm{r}^{3} \varepsilon_{\mathrm{m}} \operatorname{Re}\left\{\frac{\varepsilon_{\mathrm{p}}^{*}-\varepsilon_{\mathrm{m}}^{*}}{\varepsilon_{\mathrm{p}}^{*}+2 \varepsilon_{\mathrm{m}}^{*}}\right\} \nabla\left|E_{r m s}\right|^{2} .
$$

In this expression, $E_{r m s}$ is the root mean square amplitude of the electric field. $\varepsilon^{*}$ is the complex dielectric constant, expressed by $\varepsilon^{*}=\varepsilon+\frac{\sigma}{\mathrm{i} \omega}$, where $\varepsilon$ is the dielectric constant (subscripts $\mathrm{m}$ and $\mathrm{p}$ stand for medium and particle, respectively), $\sigma$ is the liquid conductivity, and $\omega$ the frequency of the electric field. Depending on the permittivity difference between the particle and the medium, DEP force can be either positive or negative, suggesting that nanoparticles can be either attracted to or repelled away from a greater electric field area. The essential part for the DEP force expression is in the bracket, which is known as Clausius-Mossotti (CM) factor. ${ }^{22,23}$ As plotted in Fig. 5, the CM factor is a negative constant for the PS/water system between 0.1 and $1000 \mathrm{~Hz}$, while that for the $\mathrm{TiO}_{2}$ /toluene system decreases with increasing frequency till $10 \mathrm{~Hz}$ and remains at a positive constant afterwards. Essentially, it is the numerical contrast between dielectric constant of particle and solvent that determines the properties of frequency-dependent DEP force. The parameters used in the calculation were: $\mathrm{r}=175 \mathrm{~nm}, \varepsilon_{P S}=2.6, \sigma_{P S}=1.0 \times 10^{-16} \mathrm{~S} /$ $\mathrm{m}, \varepsilon_{D I \text { Water }}=80.1, \sigma_{\text {DI Water }}=5.5 \mu \mathrm{S} / \mathrm{m}$ for PS/DI water system, and $\mathrm{r}=50 \mathrm{~nm}, \varepsilon_{\mathrm{TiO}_{2}}=86, \sigma_{\mathrm{TiO}_{2}}=0.4 \mathrm{~S} / \mathrm{m}, \varepsilon_{\text {toluene }}=2.4$, $\sigma_{\text {toluene }}=0 \mathrm{~S} / \mathrm{m}$ for $\mathrm{TiO}_{2} /$ toluene system. ${ }^{24}$

During the droplet drying process with AC signal applied, there are several factors that affect the particle motion, namely, the capillary flow, the pinning force, the surface tension force and the DEP force (Fig. 6). Capillary flow is generated inside the droplet due to a faster solvent evaporation rate at the periphery of the droplet, and it transports nanoparticles to the three phase contact line. The pinning force is
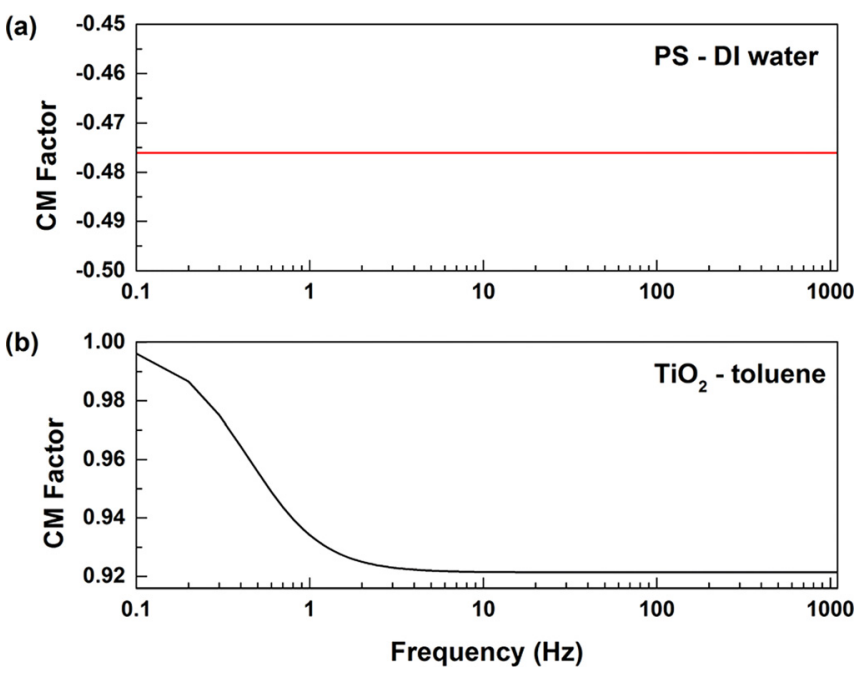

FIG. 5. Plots of the CM factor as a function of AC signal frequency for PS/ water (a) and $\mathrm{TiO}_{2}$ /toluene systems (b). The CM factor for the PS/water system is a negative constant for frequencies in the range of $0.1-1000 \mathrm{~Hz}$, but, for the $\mathrm{TiO}_{2}$ /toluene system, it decreases with increasing frequency, and stays as a positive constant starting around $10 \mathrm{~Hz}$. 

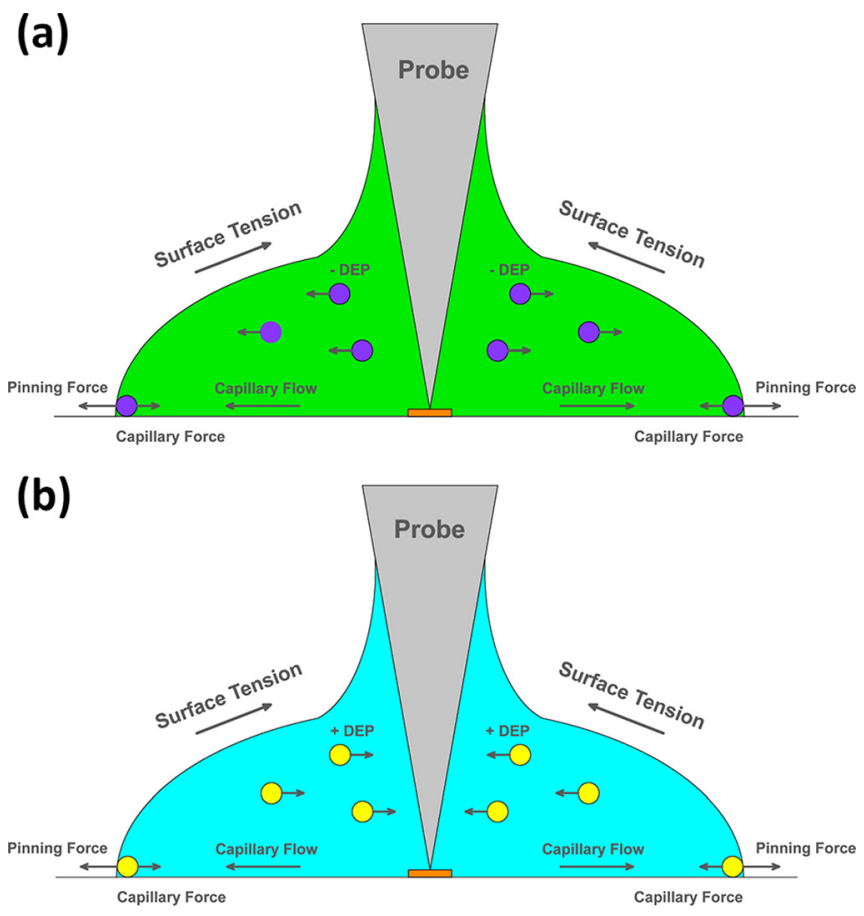

FIG. 6. Schematic illustrations of the involved forces in the process of pattern formation. (a) Profile of PS/DI water system; (b) Profile of $\mathrm{TiO}_{2} /$ toluene system. The droplets are placed on insulating substrates and within the outer electrode.

created due to irregularities at the contact line such as the accumulated nanoparticle clusters, prevents the edge of droplet from retracting, ${ }^{2}$ and is proportional to the perimeter of the contact line. $^{25}$ The outermost nanoparticles at the contact line also experience a surface tension related force that is called capillary force or de-pinning force, defined as $F_{C}=$ $2 \pi r \gamma_{l g} \cos ^{2} \theta$ ( $r$ is the radius of nanoparticles, $\gamma_{l g}$ is the surface tension at the liquid-gas interface, and $\theta$ is the contact angle). ${ }^{26}$ During the "stick" process, evaporation of solvent causes the decrease of contact angle and, thus, the increase of capillary force. When it exceeds the pinning force, the contact line is de-pinned and recedes to the next position, completing the "slip" step and leaving behind a ring of nanoparticles. 19,26 The formation of concentric rings is essentially due to the repetitive "stick-slip" cycles.

For the PS/water system in Fig. 6(a), the DEP force is negative and works together with capillary flow, driving the PS nanoparticles to the three phase contact line. On the other hand, the DEP force is independent of the AC frequency, hence the pattern formation does not vary significantly from 1 to $100 \mathrm{~Hz}$. Compared to the case without the AC signal, the larger patterned areas were actually attributed to the electro-wetting driven internal flow via excitation of eigenmodes triggered at frequencies ranging from $10 \mathrm{~Hz}$ to a few kHz. ${ }^{16,27}$ Since water does not evaporate as quickly as some organic solvents, the PS/DI water droplet keeps its maximum size for a significant fraction of total evaporation time after being placed on the substrate. ${ }^{2}$ During this period of time, this internal flow field, with maximum flow velocities of several hundred $\mu \mathrm{m} s^{-1}$, counteracts with the capillary flow and the DEP force. ${ }^{16}$ As a result, a large number of PS nanoparticles stay in the droplet to form concentric rings over a larger area instead of accumulating along the contact line. At $0.1 \mathrm{~Hz}$, the patterned area $\left(4.83 \mathrm{~mm}^{2}\right)$ is smaller than those under higher frequencies. This is likely due to the weakened internal flow field at $0.1 \mathrm{~Hz}$ : motion of PS nanoparticles are mainly dominated by the effects of capillary flow and the DEP force, resulting in a smaller patterned area.

For the $\mathrm{TiO}_{2} /$ toluene system in Fig. 6(b), however, the DEP force competes with capillary flow and attracts the $\mathrm{TiO}_{2}$ nanoparticles towards the center probe. When the AC frequency increases from 0.1 to $10 \mathrm{~Hz}$, the DEP force decreases, which means more nanoparticles have been transported by the capillary flow to the first pinned contact line near the outer electrodes (not shown in the pictures). Consequently, fewer nanoparticles remain in the droplet, and the pinning of the nanoparticles occurs when the edge of the droplets recedes to a position with a smaller perimeter, thus forming a concentric ring with reduced patterned area. The number of concentric rings decreases while the inter-ring spacing increases with the AC frequency as the contact line has to travel longer distance to the next pinning position. Interestingly, the patterned area of $\mathrm{TiO}_{2}$ nanoparticles gradually decreases even though the CM factor becomes a constant above $10 \mathrm{~Hz}$. This could be resulted from the electrostatic effect which weakened at higher frequencies: the negatively charged $\mathrm{TiO}_{2}$ nanoparticles are less likely to be attracted via electrostatic force by the center probe at higher frequencies. The electro-wetting effect is negligible in this case because toluene evaporates faster than water, and it takes shorter time for the contact line to release from the outer electrode.

It should be noted that when the drying front moves away from the outer electrode, the DEP forces might change slightly due to the variation in electric field. Better electrode design is required in the future studies to improve the control over the pattern formation while the drying front concedes.

Finally, although the AC signal was applied on the droplet during the pattern formation, it had little thermal effect because of the poor conductivity of the droplet and substrate as well as the low AC frequencies we applied. As a result, the patterning process was not disturbed by the electrothermal effect, as confirmed by the nearly constant solvent evaporation rate at different frequencies in Fig. 4(b). This observation is also consistent with a key point in a literature review by Malika Ammam, stressing that in poorly conductive solvents, the low frequency AC signals with small amplitudes contribute little to the effect of Joule heating and, hence, the electrothermal flow. ${ }^{28}$

In summary, we have demonstrated the feasibility of using AC signal to modulate the patterning of concentric rings of polystyrene and titanium dioxide nanoparticles from evaporating droplets. The spacing of the rings and the patterned areas can be manipulated by tuning the frequencies of applied AC signal. We explained the frequencydependent pattern formation based on dielectrophoresis. We believe that the AC signal guided coffee ring effect is applicable to other nanoparticles of different materials, shapes, and in different solutions. It can also be used together with other guiding conditions such as surface 
topography to pattern functional nanostructures for various device applications.

${ }^{1}$ R. D. Deegan, O. Bakajin, T. F. Dupont, G. Huber, S. R. Nagel, and T. A. Witten, Nature 389, 827 (1997).

${ }^{2}$ R. D. Deegan, Phys. Rev. E 61, 475 (2000).

${ }^{3}$ R. D. Deegan, O. Bakajin, T. F. Dupont, G. Huber, S. R. Nagel, and T. A. Witten, Phys. Rev. E 62, 756 (2000).

${ }^{4}$ P. J. Yunker, M. A. Lohr, T. Still, A. Borodin, D. J. Durian, and A. G. Yodh, Phys. Rev. Lett. 110, 035501 (2013).

${ }^{5}$ T. Still, P. J. Yunker, and A. G. Yodh, Langmuir 28, 4984 (2012).

${ }^{6}$ N. D. Denkov, O. D. Velev, P. A. Kralchevsky, I. B. Ivanov, H. Yoshimura, and K. Nagayama, Langmuir 8, 3183 (1992).

${ }^{7}$ C. D. Dushkin, K. Nagayama, T. Miwa, and P. A. Kralchevsky, Langmuir 9, 3695 (1993).

${ }^{8}$ M. Yamaki, K. Matsubara, and K. Nagayama, Langmuir 9, 3154 (1993).

${ }^{9}$ F. C. Chen, J. P. Lu, and W. K. Huang, IEEE Photonics Technol. Lett. 21, 648 (2009).

${ }^{10}$ J. T. Wen, C. M. Ho, and P. B. Lillehoj, Langmuir 29, 8440 (2013).

${ }^{11}$ T. S. Wong, T. H. Chen, X. Y. Shen, and C. M. Ho, Anal. Chem. 83, 1871 (2011).

${ }^{12}$ L. Zhang, H. Liu, Y. Zhao, X. Sun, Y. Wen, Y. Guo, X. Gao, C. Di, G. Yu, and Y. Liu, Adv. Mater. 24, 436 (2012).

${ }^{13}$ L. Cui, J. Zhang, X. Zhang, L. Huang, Z. Wang, Y. Li, H. Gao, S. Zhu, T. Wang, and B. Yang, ACS Appl. Mater. Interfaces 4, 2775 (2012).
${ }^{14}$ H. Hu and R. G. Larson, J. Phys. Chem. B 110, 7090 (2006).

${ }^{15}$ D. Orejon, K. Sefiane, and M. Shanahan, J. Colloid Interface Sci. 407, 29 (2013).

${ }^{16}$ H. B. Eral, D. M. Augustine, M. H. G. Duits, and F. Mugele, Soft Matter 7, 4954 (2011).

${ }^{17}$ D. Mannetje, F. Mugele, and D. van de Ende, Langmuir 29, 15116 (2013).

${ }^{18}$ D. Mampallil, H. B. Eral, A. Staicu, F. Mugele, and D. van de Ende, Phys. Rev. E 88, 053015 (2013).

${ }^{19}$ J. Xu, J. F. Xia, S. W. Hong, Z. Q. Lin, F. Qiu, and Y. L. Yang, Phys. Rev. Lett. 96, 066104 (2006).

${ }^{20}$ Y. J. Chen, K. Suzuki, H. Mahara, K. Yoshikawa, and T. Yamaguchi, Appl. Phys. Lett. 102, 041911 (2013).

${ }^{21}$ C. Pozrikids, Introduction to Theoretical and Computational Fluid Dynamics (Oxford University Press, New York, 2011), p. 281.

${ }^{22}$ T. B. Jones, Electromechanics of Particles (Cambridge University Press, New York, 1995), p. 36.

${ }^{23}$ H. Morgan and N. Green, AC Electrokinetics: Colloids and Nanoparticles (Research Studies Press, Philadelphia, 2002), p. 51.

${ }^{24}$ D. R. Lide, CRC Handbook of Chemistry and Physics (CRC Press, Boca Raton, 2006).

${ }^{25}$ P. G. de Gennes, Rev. Mod. Phys. 57, 827 (1985).

${ }^{26}$ B. M. Weon and J. H. Je, Phys. Rev. Lett. 110, 028303 (2013).

${ }^{27}$ F. Mugele, J.-C. Baret, and D. Steinhauser, Appl. Phys. Lett. 88, 204106 (2006).

${ }^{28}$ M. Ammam, RSC Adv. 2, 7633 (2012). 